\title{
Frequency and time properties of decimeter narrowband spikes in solar flares
}

\author{
Shujuan Wang \\ Key Laboratory of Solar Activity / National Astronomical Observatories, \\ Chinese Academy of Sciences, Beijing 100012, P. R. China \\ email: wsj@nao.cas.cn
}

\begin{abstract}
In this paper, we focus to study the frequency and time properties of a group of spikes recorded by the $1.08-2.04 \mathrm{GHz}$ spectrometer of NAOC on 27 October 2003. At the first we calculate the mean and minimum bandwidth of the spikes. We apply two different methods based on the wavelet analysis according to Messmer \& Benz (2000). The first method determines the dominant spike bandwidth scale based on their scalegram, and the second method is a feature detection algorithm in the time-frequency plane. Secondly the time profile of each single spike was fitted and analyzed. In particular, we determined the e-folding rise and decay times corresponding to the ascending and decaying parts of the time profile, respectively. Several important correlations were studied and compared with the results in earlier literature, i.e. those between duration and frequency, e-folding rise time and decay time, e-folding decay time and duration, and e-folding decay time and peak flux. Finally some parameters of source region were estimated and the possible decaying mechanism was discussed.
\end{abstract}

Keywords. Solar, flare, decimeter, spike

\section{Observations and Data Processing}

A group of radio spikes were observed by the SBRS at Huairou Station of NAOC with the super-high spectral and temporal resolutions of $4 \mathrm{MHz}$ and $5 \mathrm{~ms}$ on 27 October 2003 (Figure 1). The spikes were superposed on the continuum burst, so that we have to eliminate the continuum burst background from the total radio burst to extract the spikes. We used the method reported in our former work (Wang et al., 2002) to extract the spikes. As a result of filtering and denoising, 420 microwave spikes were extracted out. Then smoothing and fitting by a Hermite cubic spline method (Aschwanden and Benz, 1986), the time profile of spike was obtained.

\section{Discussions and Conclusions}

The first part is for frequency properties. The narrow bandwidth of spikes is a important criteria for the emission mechanisms. Generally the bandwidth of a single spike depends on two factors: (i) the intrinsic bandwidth of the emission process times (ii) the variation of the emission frequency over the source region, caused by the range of some plasma parameters. We used two different methods, based on the wavelet analysis according to Messmer \& Benz (2000), to measure the minimum and the mean bandwidth of spikes statistically. For the first method, the scalegrams of spike burst (A) and background (B) were estimated. If the scalegram values of A and B began to apart, the minimum bandwidth could be measured; if the scalegram values of $\mathrm{A}$ exceeded the $3 \delta$ level of noise scalegram of $\mathrm{B}$, the mean bandwidth could be measured. For the second method, after the denoising procedure, a Gaussian was fitted into every single spike profile. The mean and minimum bandwidth of the spikes could be measured statistically. 


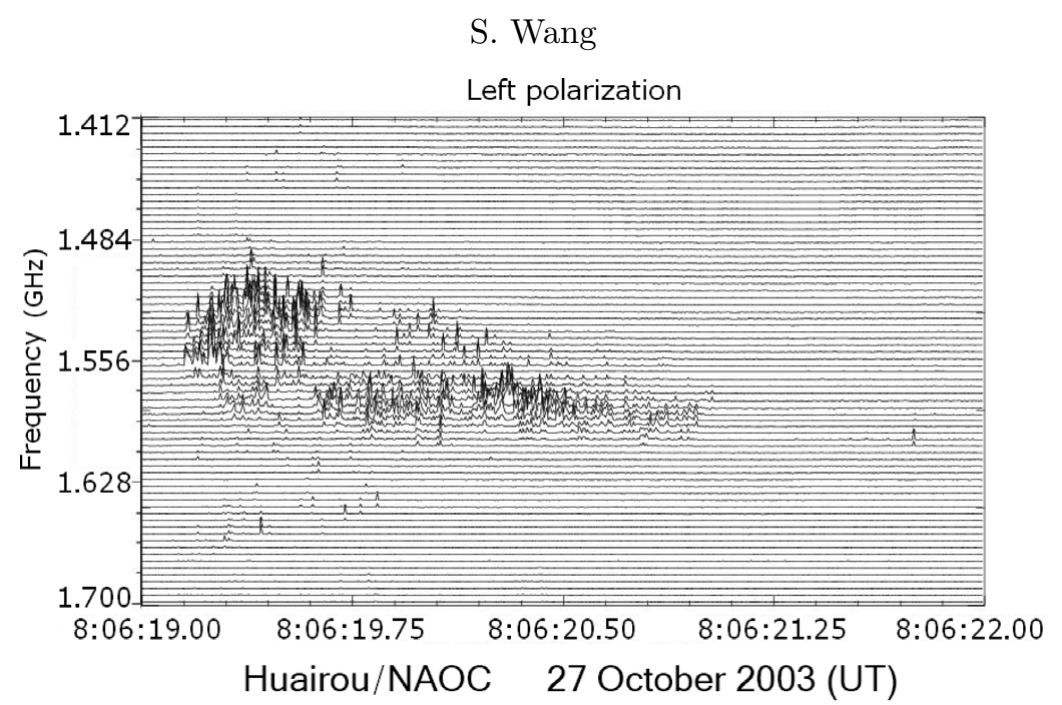

Figure 1. Spectra of the cluster of spikes on 27 Oct. 2003

Results: the mean and minimum bandwidth of the spikes were measured as $16 \mathrm{MHz}$ and $8 \mathrm{MHz}$ by the first method and $16.3 \mathrm{MHz}$ and $4.7 \mathrm{MHz}$ by the second method. The minimum bandwidth sets the most severe requirements on the emission process and limits the source size.

The second part is for time properties. According to the research of Güdel et al. (1990), assume an exponential function to fit the ascending and decaying phase of spikes, it as $f(t)=C \exp (t / \tau)+f_{0}$. Then we could obtained the relationship of $1 / \tau=\mathrm{d}(\ln (f(t)-$ $\left.f_{0}\right)$ )/dt, where $f(t)$ refers to the observational flux of spikes, $f_{0}$ to the background flux, $\tau$ to the e-folding times $\tau_{a}$ (rise) or $\tau_{d}$ (decay), $C$ to a fitting constant. Statistic results gave out that there were 73 spikes appeared ascending or decaying exponentially. we found the dispersion between $\tau_{a}$ and $\tau_{d}$ was broad a few. In other words, this event suggested that the time profile of spike is asymmetry generally.

Then we do statistics of $\tau_{d}$ vs. duration and $\tau_{d}$ vs. peak flux. From the results of statistics, it was difficult to find the correlation between $\tau_{d}$ and the duration, or between $\tau_{d}$ and the peak flux of the spikes. Therefore it should be suggested that the decay process could be caused by plasma collision.

We also calculated the electron temperatures $T$ using the conventional formula for electron-ion collisions (Güdel et al., 1990 ): $T=8.1 \times 10^{3} \nu^{4 / 3} \tau_{d}^{2 / 3}$, where $\nu$ in $\mathrm{MHz}, \tau_{d}$ in $\mathrm{s}$ and $T$ in $\mathrm{K}$. It was found that most of the temperatures were in the magnitude of $10^{6} \mathrm{~K}$, comparing to typical temperature of the coronal plasma. Therefore the plasma hypothesis could be a plausible explanation for the exponential decay of spike.

Acknowledgments. This work is supported by NSFC Grants No. 10921303 and 10903013, MOST Grant No. 2011CB811401, the National Major Scientific Equipment R\&D Project ZDYZ2009-3.

\section{References}

Aschwanden, M. J. \& Benz, A. O. 1986, A\&A, 158, 102.

Güdel, M. \& Benz, A. O. 1990, A\&A, 231, 202.

Messmer, P. \& Benz, A. O. 2000, A\&A, 354, 286.

Wang, S., Yan, Y., \& Fu, Q. 2002, Solar Phys., 209, 185. 